\title{
Utilisation de rubans de plastique pour la récolte des œufs et des stades larvaires et nymphaux de Simulies (Diptères Paranématocères) et pour l'étude biologique de ceux-ci.
}

\author{
par J.-M. DOBY, B. RAULT et F. BEAUCOURNU-SAGUEZ
}

La récolte des stades aquatiques de Simulies n'est pas toujours facile. Les œufs sont disposés, en amas souvent, isolément parfois, sur les feuillages qui pendent des berges et traînent dans les zones de courant. Les larves et nymphes s'observent fixées sur ces mêmes feuillages, sur les branches, ainsi que sur les pierres et sur la végétation immergées.

L'utilisation de supports artificiels pour cette récolte est relativement récente (1). Les premiers, Wolfe et Péterson (1958), ont utilisé des supports constitués par des cônes de métal peints en blanc et fixés aux pierres par des fils de fer. L'année suivante, ces mêmes auteurs utilisèrent des morceaux de bois, cylindres ou morceaux de planches carrés, maintenus à différentes profondeurs par des cordes dans les zones de courant.

De même, en 1962, Carlsson récolta d'une façon satisfaisante les stades aquatiques d'un certain nombre d'espèces sur des surfaces planes constituées par des planches, des morceaux de vitres, fixés par des pierres dans le courant au fond du lit de ruisseaux.

Toutefois, l'examen des supports naturels révèle que les stades aquatiques de la plupart des espèces de Simulies se fixent de préférence sur les végétaux dont les feuilles longues et rubanées ondulent dans les zones de courant. Il était donc logique d'utiliser de préférence comme supports artificiels un matériel rappelant d'assez près ce type de végétation.

En 1962, Williams et Obeng utilisèrent, en même temps d'ailleurs que des entonnoirs en polythène qui ne leur donnèrent que de médiocres résultats, des rubans, de $2,5 \mathrm{~cm}$ de large et de $50 \mathrm{~cm}$ environ de long taillés dans des feuilles de polythène. Ces rubans

(1) On ne peut considérer comme support vraiment artificiel le matériel utilisé par plusieurs chercheurs russes (Shipitzina et coll.-1959, Zviaguintzev-1961, Timofééva-1962) pour suivre l'efficacité de certaines substances insecticides sur les larves de simulies et la rapidité du repeuplement, matériel consistant en petits balais de rameaux de saules attachés sur une corde à différentes profondeurs, une des extrémités de la corde étant lestée au fond et l'autre attachée à une poutre de bois flottante ou à une bouée métallique. 

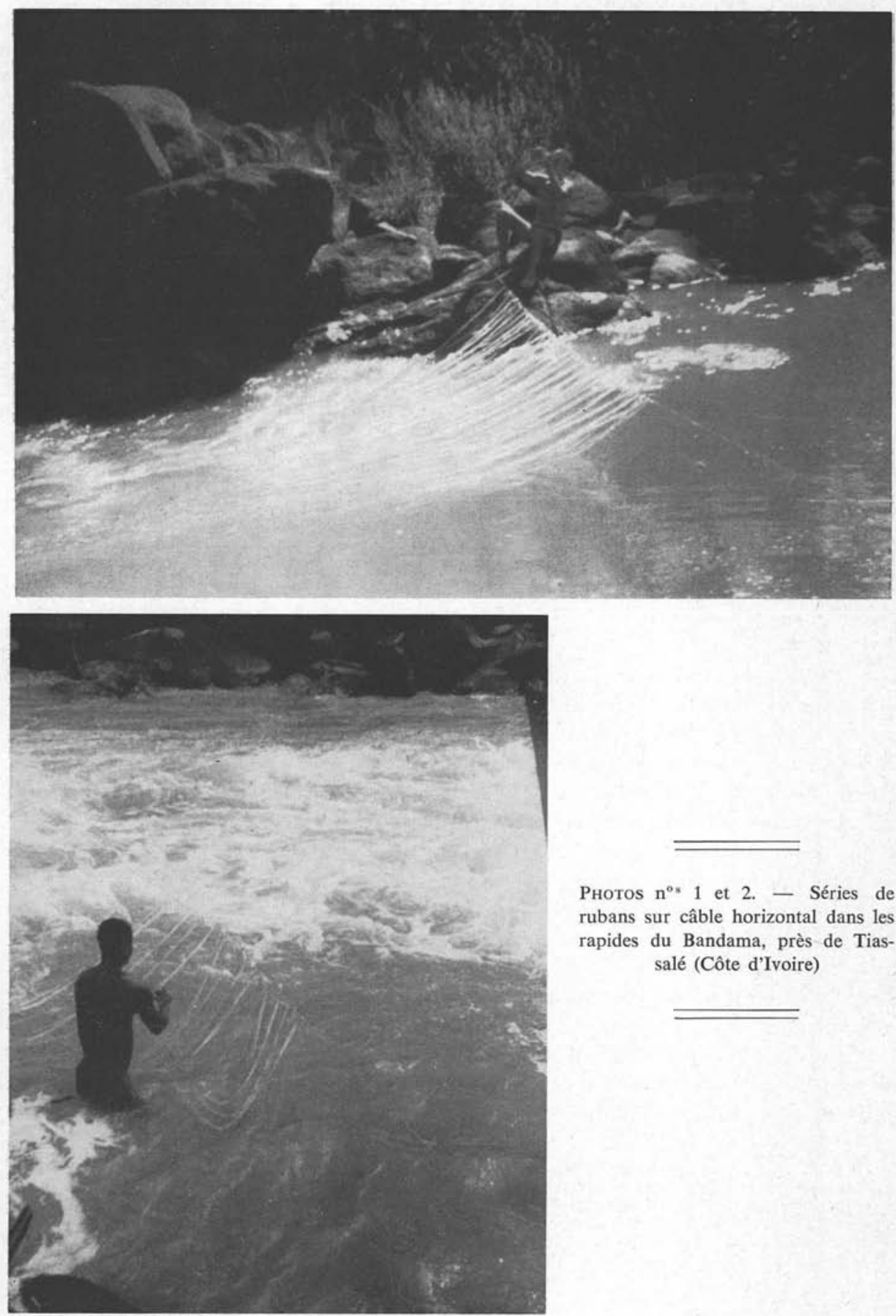

Pнотоs $\mathrm{n}^{\circ *} 1$ et 2 . - Séries de rubans sur câble horizontal dans les rapides du Bandama, près de Tiassalé (Côte d'Ivoire) 


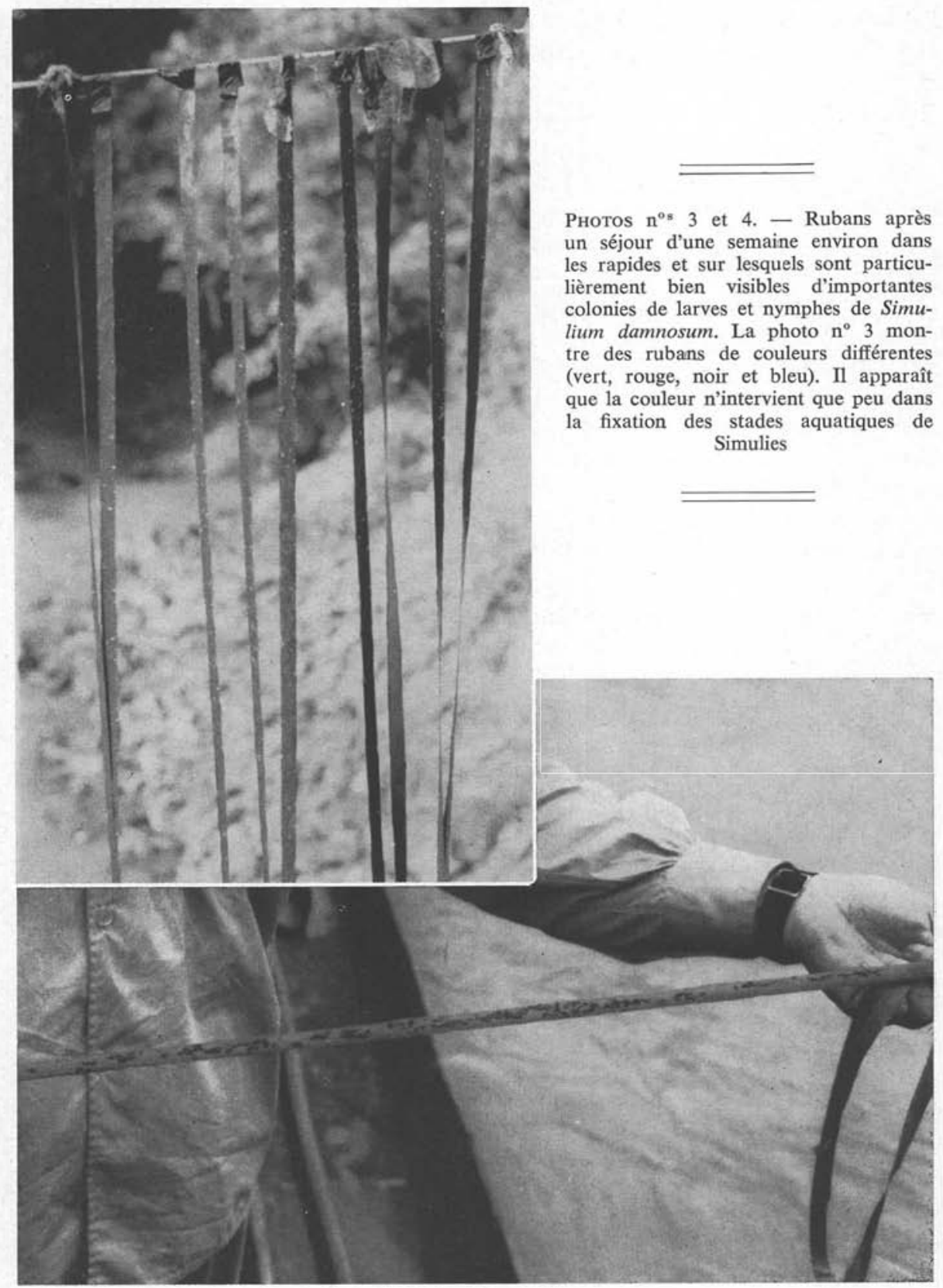


étaient attachés un par un par une de leurs extrémités aux petites pierres et traînaient dans le courant. D'autres rubans étaient fixés par une extrémité au centre de morceaux de bois de $15 \mathrm{~cm}$ de long environ coincés entre les pierres du fond. Ces deux auteurs disent avoir obtenu ainsi de bons résultats, mais signalent que ces rubans isolés sont peu visibles dans le courant et difficiles à retrouver.

Nous avons repris l'idée de Williams et Obeng, mais nous utilisons des rubans fixés par séries sur une cordelette, selon les techniques ci-dessous décrites, ce qui permet de récolter des larves et des nymphes pratiquement sur toute la largeur d'un cours d'eau donné et à n'importe quelle profondeur.

La prospection systématique d'un cours d'eau n'est possible que lorsque celui-ci a des dimensions très réduites tant en profondeur qu'en largeur. Quand celles-ci dépassent certaines valeurs, on éprouve alors souvent de grandes difficultés pour la récolte des stades aquatiques de simulies. Beaucoup des supports possibles ne peuvent en effet être atteints des berges. Souvent, la rapidité du courant, la présence de remous sur roches rendent dangereux et parfois impossible l'accès en bateau des supports éloignés du bord. Même quand les ruisseaux prospectés sont de petites dimensions, à certaines périodes de l'année, l'accès des supports habituels situés dans les zones de courant est également impossible en raison de l'envahissement des berges par les eaux en crue.

Un problème analogue se pose lorsque la profondeur dépasse quelques dizaines de centimètres. S'il est possible, bien que cela s'effectue souvent avec difficultés, de remonter à la surface la végétation poussant sur les fonds, les stades fixés en profondeur sur des rochers ou sur des pierres volumineuses sont impossibles à atteindre. Or, si les stades aquatiques de beaucoup d'espèces se fixent classiquement sur des supports à quelques centimètres seulement sous la surface de l'eau, ceux de certaines, par contre, sont susceptibles de se maintenir à grande profondeur (2).

De plus, lorsqu'il s'agit de stades fixés en profondeur, même lorsqu'il est possible de les ramener à la surface, il est presque toujours impossible de savoir à quelle profondeur exacte ils étaient.

Les larves de Simulies étant susceptibles d'importantes migrations d'un support sur un autre, soit volontairement en raison de modifications localisées de courant ou de hauteur d'eau, soit involontairement à la suite de décrochage et d'entraînement par le courant, nous avons pensé utiliser la tendance qu'elles ont à se refixer de préférence sur des supports végétaux allongés et ondulant entre deux eaux, comme il a été dit plus haut.

Au cours d'un séjour en 1965, en Afrique, nous avions utilisé dans ce but, comme supports, respectivement des bandes de toile, de gaze, de cellophane et des ficelles et cordages de natures diverses. Toutefois, ce matériel ne nous donna absolument pas satisfaction en raison, soit de sa putréfaction rapide, soit de sa grande facilité à se déchirer et à se rompre sous l'action du courant. En effet, la putréfaction de certaines de ces matières, due au développement des bactéries, était alors d'autant plus rapide qu'il s'agissait d'essais dans une eau particulièrement chaude, aux environs de 30 à $35^{\circ}$.

(2) Par exemple, des larves et nymphes de Simulium columbaschense ont pu être récoltées dans le Danube à plus de $20 \mathrm{~m}$ de profondeur. 
En 1966, nous avons donc choisi un matériel constitué par des rubans de $150 \mathrm{~cm}$ de long sur 1,5 à $2 \mathrm{~cm}$ de large, en une matière plastique suffisamment mince pour permettre une souple ondulation sous l'action du courant. Ce matériel a été testé avec succès dans les rapides du fleuve Bandama, en Côte d'Ivoire, et s'est révélé suffisamment résistant et absolument imputrescible, ce qui a permis le maintien dans l'eau pendant plusieurs semaines sans altération.

Nous avons utilisé des rubans de plastique de différentes couleurs. Nous ignorions en effet, au départ, si, en dépit de l'immersion parfois à grande profondeur, la coloration pouvait avoir une influence sur la fixation des larves et la formation des nymphes. L'expérience a montré que ce facteur était sans importance décelable. Aussi, est-il préférable d'utiliser des rubans de teintes claires, qui sont facilement repérables dans la végétation ou entre deux eaux. La présence des larves et nymphes, qui sont généralement sombres, y est également plus facilement décelée à une certaine distance et même sous plusieurs centimètres d'eau.

Nous avons choisi comme matière première un plastique en feuilles, non lisse, dont la surface finement granitée présente des microalvéoles, ce qui facilite, pensons-nous, la fixation des larves. Il s'agissait de feuilles utilisées habituellement pour couvrir les cahiers et livres d'école.

Les rubans ont été utilisés soit isolément, comme l'avaient fait Williams et Obeng, soit surtout en séries, lorsque nous avons eu affaire à des cours d'eau de grande largeur. Ces rubans, dont le nombre variait selon l'importance de la zone à étudier, étaient fixés par une de leurs extrémités sur un câble léger en nylon, c'est-à-dire en matière également imputrescible. Sur ce câble, les rubans étaient séparés les uns des autres par quelques centimètres.

Ces rangées étaient installées horizontalement en travers des cours d'eau dont la zone médiane était inaccessible par bateau, en raison, par exemple, de la violence du courant (rapides et chutes des fleuves africains). Leur pose n'est possible qu'à condition toutefois que la largeur permette de faire' franchir le cours d'eau au câble de soutien (entraînement par jets de pierres ou tirs de flèches d'arc).

La présence d'une boucle de coulissage sur le point d'attache de l'une des berges permet, à condition d'avoir un câble de soutien assez long, le retour de celui-ci sans avoir, après la première installation, à traverser une nouvelle fois un fleuve parfois difficile. On peut ainsi ramener à soi, d'un bord à l'autre, les larves et nymphes vivant au milieu du cours d'eau, et de remettre éventuellement immédiatement l'ensemble du matériel en position.

Dans certains cas, toutefois, la largeur du fleuve à prospecter ne permet pas de tendre, du moins avec les moyens dont dispose habituellement l'entomologiste sur le terrain, un câble d'un bord à l'autre.

La technique suivante permet alors d'explorer cependant une grande partie de la largeur du cours, notamment les zones trop éloignées des bords pour être normalement directement accessibles à la prospection.

Les séries de rubans sont alors fixées sur un câble dont l'une des extrémités est lestée avec une grosse pierre lancée loin dans le courant. La fixation de l'autre extrémité 
peut se faire, soit sur un élément fixe de la berge (rochers, souches, troncs d'arbres), soit sur une bouée flottante, elle-même reliée à la berge par un câble.

Ici aussi, l'existence d'une boucle de coulissage sur la pierre de lestage et d'une longueur suffisante de câble permet de ramener à soi les supports pour examen, tout en laissant en place la pierre et de les replacer ensuite rigoureusement dans la même situation.

La connaissance de la position d'attache d'un ruban déterminé, repéré par numérotage par exemple, dans la série fixée sur le câble, et la mesure de l'angle de sortie de celui-ci hors de l'eau, mesure facilement effectuée avec un simple rapporteur et un

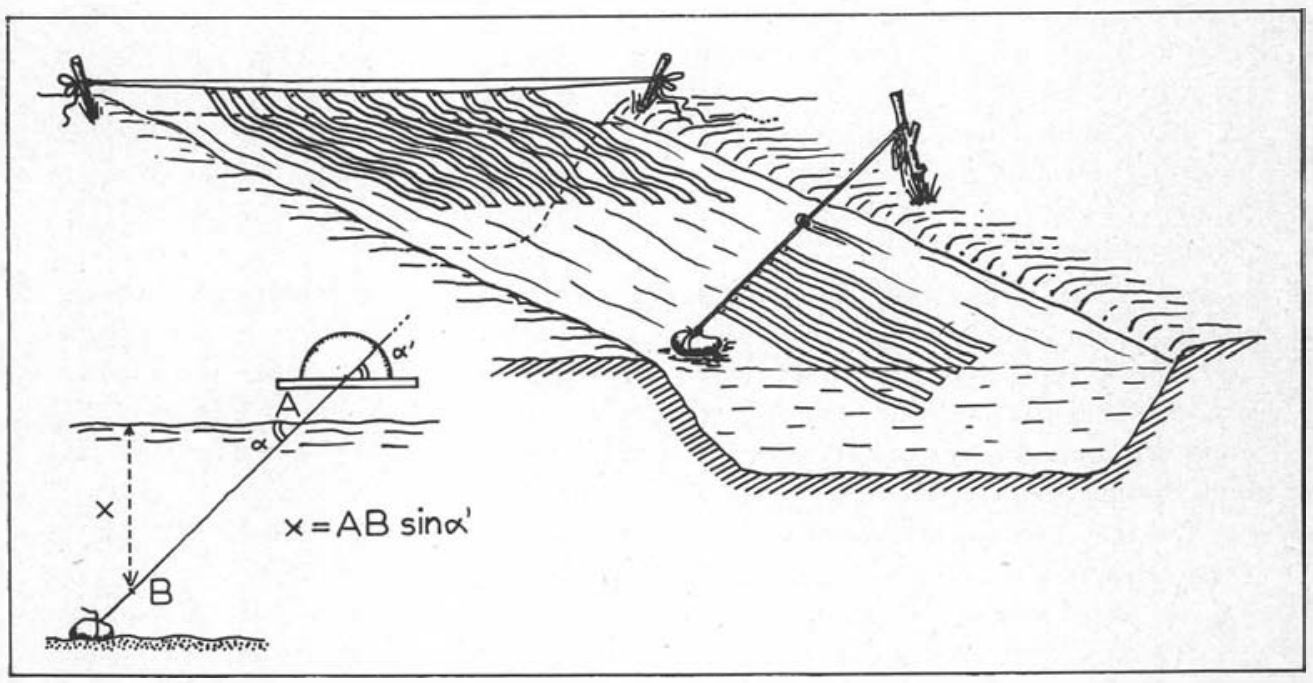

niveau d'eau, permet de calculer la profondeur où se trouvait le ruban précité, et, par conséquent, celle à laquelle les larves et les nymphes s'y trouvaient fixées. On a, en effet, la relation suivante :

$x$ (profondeur où se trouvait le ruban) $=\mathrm{AB} \sin \alpha$ ( $\mathrm{AB}$ étant la longueur de câble entre le point de fixation du ruban et le point d'émergence du câble hors de l'eau et $\alpha$ étant l'angle formé par le câble et l'horizontale).

La technique des rubans, utilisée sous une forme ou sous l'autre, permet d'étudier de nombreux points de la biologie des stades aquatiques des Simulies:

- répartition en profondeur, migration, répartition dans le temps des colonies et étude des stades qui les composent, surveillance pendant plusieurs mois des résultats d'opération de désinsectisation, etc... 


\section{Bibliographie}

Carlsson (G.), 1962. - Studies on Scandinavian black flies. Opuscula entomologica. suppl. 21, Lund, Berlingska Boktryckeriet.

SHIPItZina et coll., 1959. - In Timofeeva et coll., 1962.

Timofeeva (L.-V.), Mitrofanov (A.-M.), Markovic (N.-I.), Muraviéva (T.-V.), Shankov (M.-E.) et Tupitzine (L.-F.), 1962. - [Essai couronné de succès de lutte contre les moucherons buveurs de sang (Diptera, Simuliidae) par le traitement des lieux de reproduction] (en Russe). Meditzinskaïa Parazitologina et parazitnye Bolezni, Moscou, 3.

Williams (T. R.) et OBeng (L.), 1962. - A comparison of two methods of estimating changes in Simulium larvae populations, with a description of a new method. Ann. Trop. Med. a. Parasit., 56, 359.

Wolfe (L. S.) et PÉterson (D. G.), 1958. - A new method to estimate levels of infestations of black-fly larvae (Diptera : Simuliidae). Canad. Jl of Zool., 36, 863.

-, - 1959. - Black-flies (Diptera : Simuliidae) of the forests of Quebec. Canad. Jl of Zool., $37,152$.

ZviaguintZev, 1961. - In Timoteeva et coll., 1962.

Laboratoire de Parasitologie et Zoologie appliquée de la Faculté de Médecine et de Pharmacie de Rennes, et Station d'Ecologie tropicale de Lamto (Directeur, $\mathrm{P}^{\mathrm{r}}$ M. Lamotte, Tiassalé, Côte d'Ivoire). 